\title{
Determination of the contributions of degenerate and non-degenerate two-photon absorption response in silicon avalanche photodiode for infrared photon detection
}

\section{Guangjian Xu}

East China Normal University

\section{Xinyi Ren}

East China Normal University

\section{Qucheng Miao}

East China Normal University

Ming Yan

East China Normal University

Haifeng Pan

East China Normal University

\section{Xiuliang Chen}

East China Normal University

\section{Guang Wu}

East China Normal University

EWu ( $\nabla$ ewu@phy.ecnu.edu.cn )

East China Normal University

\section{Research}

Keywords: degenerate and non-degenerate two-photon absorption, infrared photon detection, silicon avalanche photodiode

Posted Date: March 23rd, 2020

DOI: https://doi.org/10.21203/rs.3.rs-18288/v1

License: (c) (1) This work is licensed under a Creative Commons Attribution 4.0 International License. Read Full License 
Version of Record: A version of this preprint was published at Journal of Modern Optics on September 19th, 2020. See the published version at https://doi.org/10.1080/09500340.2020.1842926. 
Determination of the contributions of degenerate and non-degenerate two-photon absorption response in silicon avalanche photodiode for infrared photon detection

Guangjian $\mathrm{Xu}^{1}$, Xinyi Ren ${ }^{1}$, Qucheng Miao ${ }^{1}$, Ming Yan $^{1}$, Haifeng Pan $^{1}$, Xiuliang Chen ${ }^{1}$, Guang $\mathrm{Wu}^{1}$, and $\mathrm{E} \mathrm{Wu}^{1,2^{*}}$

1. State Key Laboratory of Precision Spectroscopy, East China Normal University, Shanghai 200062, China

2. Collaborative Innovation Center of Extreme Optics, Shanxi University, Taiyuan, Shanxi 030006, China

*Corresponding author: ewu@phy.ecnu.edu.cn 
Abstract:

The study of non-linear interactions in semiconductor photo-electronic devices to achieve effective and fast photon identification is an ongoing and important task. We investigated the specific contribution of degenerate and non-degenerate two-photon absorption (D-TPA and ND-TPA) response in silicon avalanche photodiode (Si-APD) for infrared photon detection at room temperature. We experimentally demonstrated that when the two laser pulses overlapped, the average D-TPA quantum detection efficiencies at $1800 \mathrm{~nm}$, and that at $1550 \mathrm{~nm}$ were measured as $3.3 \times 10^{-16}$ counts $\bullet$ pulse/photon ${ }^{2}, 4.3 \times 10^{-15}$ counts $\bullet$ pulse/photon ${ }^{2}$, respectively. And the ND-TPA quantum detection efficiency of $1800 \mathrm{~nm}$ and $1550 \mathrm{~nm}$ was measured to be $7.9 \times 10^{-16}$ counts $\bullet$ pulse/photon ${ }^{2}$. This study provides a solution for the practical infrared photon detection devices based on TPA effect in Si-APDs.

Keywords: degenerate and non-degenerate two-photon absorption, infrared photon detection, silicon avalanche photodiode.

\section{Introduction}

Since the invention of the transistor more than half a century ago, semiconductor devices have been revolutionizing our daily lives, such as computers, smartphones, virtual reality devices and so forth. The photo-electronic devices such as photodetectors have become increasingly important by supporting various modern techniques. And recently, non-linear optical devices begin to develop vigorously to convert the infrared light to the visible regime or vice versa because the main information carrier in the 
optical cable network is in the infrared but detectors of infrared light is not as good as its counterpart for the visible regime [1,2]. Especially, in the fiber-based quantum cryptography where the information is encoded with single photons at the telecom wavelengths, different infrared single-photon detectors are developed including singlephoton frequency upconversion detectors [3-5]. Moreover, non-linear optical devices based on high-order transitions between energy levels such as two-photon absorption (TPA) effect enables ultra-fast signal processing [6-8].

Non-linear optical equipment are commonly based on frequency-upconversion or TPA effect to achieve rapid infrared light signal processing and detection. The frequency upconversion scheme is based on the sum-frequency generation process where the wavelength of the signal photons is transformed into the shorter wavelength region with the preservation of all the quantum characteristics and the upconverted photons finally impinge on the active area of a silicon-based detector for detection $[9,10]$. However, in the frequency upconversion system, special nonlinear crystals are designed and cut to fulfill the phase-matching conditions by adjusting the tilting angle and the operation temperature to maintain the momentum conservation for a high conversion efficiency [11-14]. At the same time, the TPA effect caused by the free electron in a semiconductor photodetector that absorbs two photons simultaneously, thereby achieving a transition from the ground state to the excited state and releasing a photoelectron by returning to the ground state can also be used for the detection of infrared photons $[15,16]$. For example, in a Si-avalanche photodiode (Si-APD) or other detector, the electron-hole pairs are created by TPA of the infrared photons and give 
rise to a photo-avalanche pulse output directly $[17,18]$. Infrared photon detection based on TPA effect has many advantages over the frequency upconversion scheme since the TPA effect is not constrained by the phase matching, and can provide a wide operating spectral range, making the TPA scheme cheap and easy to integrate with other devices. In addition, the induced photocurrent of TPA is almost insensitive to the polarization state of the incident light.

In the TPA process, if the energies of the two absorbed photons are the same, it is the so-called the degenerate two-photon absorption (D-TPA), otherwise it is the socalled the non-degenerate two-photon absorption (ND-TPA). The TPA effect of semiconductors has been studied in different materials and structures. It has been experimentally demonstrated that the TPA effect can lead to an enhancement factor up to $100-1,000$ in direct-bandgap semiconductors [6]. The extremely ND-TPA in GaN photodiodes is utilized to perform scanned imaging of three-dimensional structures by infrared femtosecond laser pulse illumination [19]. The TPA effect in the commercial In $0.81 \mathrm{Ga} 0.19 \mathrm{As}$ photodetector has been investigated to extend the nonlinear optical response to $4500 \mathrm{~nm}$ [20]. In addition, the nanostructured indium phosphide (InP) photodiode has been designed to detect the infrared light at room temperature using ND-TPA, and owing to the nanostructure, the photocurrent shows a gain of 24 with respect to the bulk response of InP [21]. The TPA effect has also been considered in silicon materials. For instance, a full band structure of TPA in bulk silicon has been theoretically calculated [22], and the D-TPA and Kerr coefficient of bulk Si for wavelength at $850 \mathrm{~nm}$ to $2200 \mathrm{~nm}$ has been measured in the experiment [23]. But the 
specific contribution value of D-TPA and ND-TPA effects in silicon photodetectors for infrared light detection has not been investigated so far as we known.

In this paper, we determined the contribution of D-TPA and ND-TPA effects for infrared light detection in the Si-APD single-photon detector at room temperature. When two beams of different wavelengths were casting on the active area of the detector with temporal separation, we got that the quantum detection efficiency of DTPA at $1800 \mathrm{~nm}$ and $1550 \mathrm{~nm}$ was about $3.2 \times 10^{-16}$ counts $\bullet$ pulse/photon ${ }^{2}$ and $4.8 \times 10^{-15}$ counts•pulse/photon ${ }^{2}$, respectively. And when the two pulses coincided in time and space, the average quantum detection efficiency for the ND-TPA of 1800 and $1550 \mathrm{~nm}$ was measured as $7.9 \times 10^{-16}$ counts $\bullet$ pulse/photon ${ }^{2}$. This research has further broadened our understanding of the TPA effect in the silicon-based photo-electronics devices, and will promote the development of infrared photon detection via TPA in Si-APDs in extended applications.

\section{Methods and Experimental}



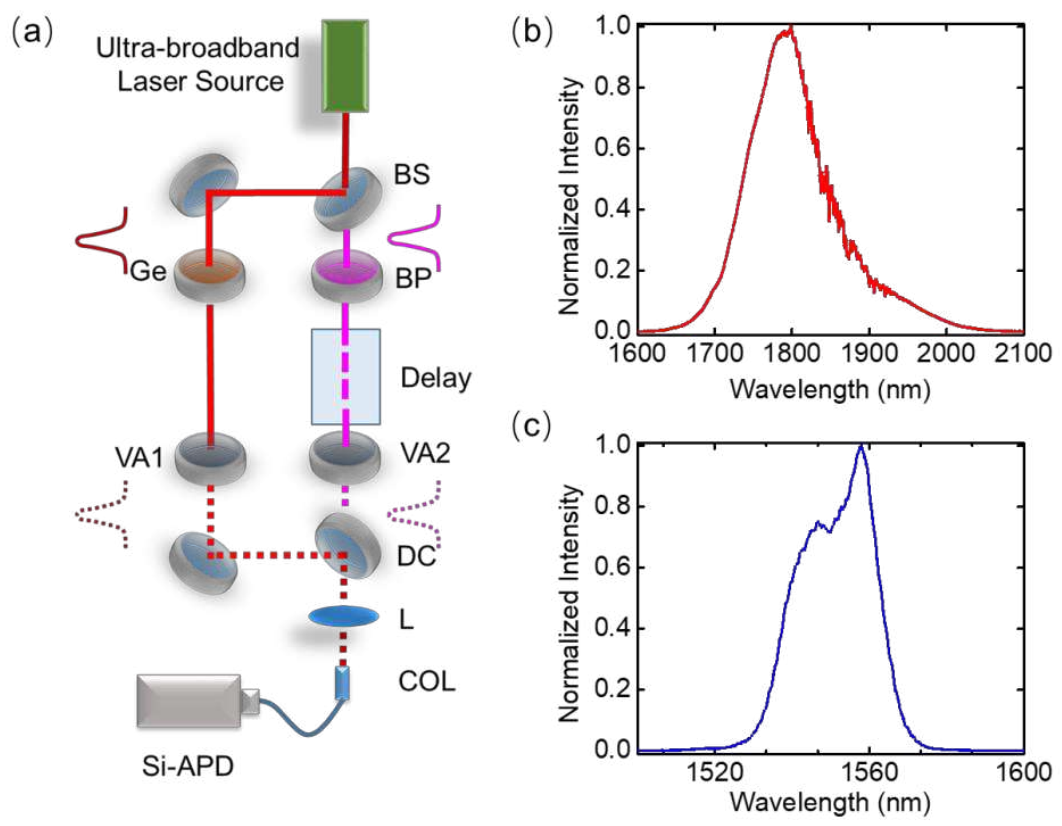

Fig. 1. (a)Experimental setup. BS: Beamsplitter; Ge: Germanium window; BP: Bandpass filter at $1550 \mathrm{~nm}$; VA1,2: Variable intensity attenuators; DC: Dichroic mirror reflecting $1800 \mathrm{~nm}$ and transmitting $1550 \mathrm{~nm}$; L: Lens, COL: Fiber collimator; Si-APD: Single-photon detector based on silicon avalanche photodiode. (b) Spectrum of the Beam A at $1800 \mathrm{~nm}$. (c) Spectrum of the Beam B at $1550 \mathrm{~nm}$.

In the experiment, we used an ultrabroadband fiber laser to study the D-TPA and NDTPA induced photon counting in the Si-APD. The experimental setup is sketched in Fig.1 (a). The spectrum of the ultrabroadband fiber laser covered from $1200 \mathrm{~nm}$ to 1900 $\mathrm{nm}$. The laser was passively mode-locked at a repetition rate of $55 \mathrm{MHz}$ with a $10 \mathrm{ps}$ pulse duration. The average output power was up to the $\mathrm{mW}$ level. A beam splitter was used to split the output laser into two beams. In one beam (Beam A), we used a germanium window to select the spectrum around $1800 \mathrm{~nm}$ as shown in Fig.1(b), while in the other beam (Beam B), a band-pass filter was inserted to select the the spectrum 
around $1550 \mathrm{~nm}$ as shown in Fig.1(c). Two variable intensity attenuators were placed in each beam to adjust the incident photon fluxes. Then the two beams were combined by a dichroic mirror, and coupled into a fiber collimator by a lens. The photons from the combined beam were captured by a fiber-coupled single-photon detector based on Si-APD (SPCM-780-14-FC, Excelitas Tech.). The diameter of the active area of the SiAPD was about $180 \mu \mathrm{m}$. The photon flux incident to the Si-APD was evaluated according to the laser power and the transmittance loss of the two beams independently.

The photon-active area of the Si-APD functioned by the p-n junction of silicon, which band gap energy is $1.12 \mathrm{eV}$. According to the wavelengths of the two in beams in our experiment, we have

$$
\hbar \omega_{A}, \quad \hbar \omega_{B}<E_{g}<2 \hbar \omega_{A}, 2 \hbar \omega_{B}
$$

where the $E_{g}$ is the bandgap energy, $\omega_{A}$ and $\omega_{B}$ are the optical frequencies of Beam A and Beam B, respectively. Because both photon energies were lower than the band gap of silicon, it is impossible for the Si-APD to effectively respond those infrared photons over $1100 \mathrm{~nm}$ by the single-photon absorption. Therefore, the Si-APD has to absorb at least two photons to trigger a photo-induced avalanche output pulse, indicating the occurrence of the two- or three-photon absorption effects. But the efficiency of threephoton absorption is much smaller than that of the TPA. So we can attribute the detection of the infrared photons in Si-APD to the TPA effect alone. Since the two beams of different optical frequencies were incident on the Si-APD simultaneously, besides the D-TPA, the ND-TPA may also happen. 


\section{Result and discussion}

In the semiconductor, the ND-TPA effect can be described according to the coupledwave equation [24]

$$
\begin{aligned}
& \frac{d I_{1}}{d z}=-2 \alpha_{2}\left(\omega_{1} ; \omega_{2}\right) I_{1} I_{2}, \\
& \frac{d I_{2}}{d z}=-2 \alpha_{2}\left(\omega_{1} ; \omega_{2}\right) I_{1} I_{2},
\end{aligned}
$$

where $I_{1}$ and $I_{2}$ are the intensities of the incident light at the optical frequencies of $\omega_{1}$ and $\omega_{2}$, respectively. And the TPA coefficient $\alpha_{2}$ is governed by the scattering matrix formalism with two parabolic bands in Ref [24]

$$
\alpha_{2}\left(\omega_{1} ; \omega_{2}\right)=K \frac{\sqrt{E_{P}}}{n_{1} n_{2} E_{g}^{3}} F_{2}\left(\frac{\hbar \omega_{1}}{E_{g}} ; \frac{\hbar \omega_{2}}{E_{g}}\right),
$$

where $E_{\mathrm{p}}$ is the Kane energy parameter, $E_{\mathrm{g}}$ is the bandgap energy, $n_{1}, n_{2}$ are the refractive indices of the material at $\omega_{1}$, and $\omega_{2}$, respectively, and $K$ is a material independent parameter. And the function $F_{2}$ is written as

$$
F_{2}\left(x_{1} ; x_{2}\right)=\frac{\left(x_{1}+x_{2}-1\right)^{3 / 2}}{2^{7} x_{1} x_{2}^{2}}\left(\frac{1}{x_{1}}+\frac{1}{x_{2}}\right)^{2} .
$$

Firstly, we studied the D-TPA in Si-APD, which means $\omega_{1}=\omega_{2}$ in Eq.(1). In the experiment, the two beams were incident on the Si-APD independently and we recorded the photon-counting rate as a function of the incident photon flux of Beam A and Beam B as shown in Fig.2. In a semiclassical approach, the photon counting rate of the SiAPD depends on the square of the incident photon flux as 


$$
C=\eta\langle N\rangle^{2}
$$

where $\eta$ represents the quantum detection efficiency by the TPA effect and $\langle N\rangle$ is the photon flux of the incident light.

(a)

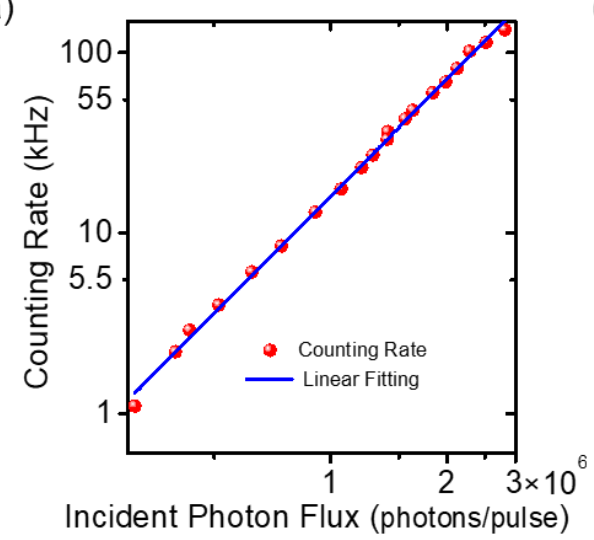

(b)

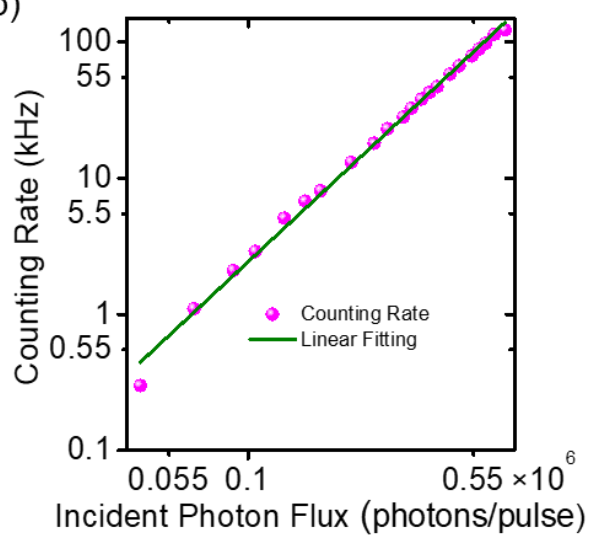

Fig.2 Photon-counting rate as a function of the incident photon flux with a single beam at $1800 \mathrm{~nm}$ (a) and $1550 \mathrm{~nm}(\mathrm{~b})$, respectively.

In D-TPA, the TPA response is a quadratic function of the incident light intensity. The slope of the logarithmic plots are 2.08 and 2.16 in Fig.2 (a), and (b) respectively, which shows that the detector's response depends quadratically on the incident photon flux both at $1800 \mathrm{~nm}$ and $1550 \mathrm{~nm}$. We can conclude that the Si-APD's response at these two wavelengths is dominated by the TPA effect. But note that in the measurement at $1800 \mathrm{~nm}$, the incident photon flux ranges from $0.3 \times 10^{6}$ to $2.8 \times 10^{6}$ photons/pulse. Meanwhile, in the measurement at $1550 \mathrm{~nm}$, the incident photon flux ranges from $0.06 \times 10^{6}$ to $0.7 \times 10^{6}$ photons/pulse. The photon counting rates were at the same level. The big difference is caused by the TPA coefficient $\alpha_{2}$ of the Si-APD at the two wavelengths. 


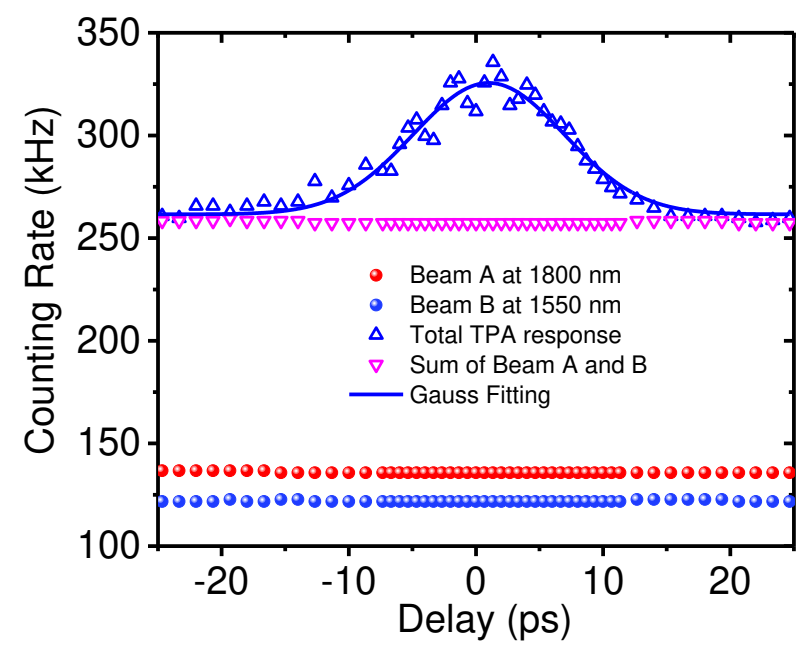

Fig.3 Photon counting rate as a function of the relative delay between the two beams. The red spots and the blue spots are the counting rate of the D-TPA of $1800 \mathrm{~nm}$ and $1550 \mathrm{~nm}$ respectively. The pink triangle spots are the sum of the both D-TPA effect. And the blue triangle spots are the counting rate of the TPA of two beams.

Then, we tuned the VAs to adjust the photon counting rate of Beam A and B at about $135 \mathrm{kHz}$ and $125 \mathrm{kHz}$, respectively. Both beams were injected to the detector but the pulse trains from the two were with a temporal delay much longer than the laser pulse duration. As shown in Fig.3, the two endpoints of the total photon counting rate represented by the blue triangles are the same as the sum from the two beams by the purple inverted triangles, showing that the Si-APD's response was from the TPA of each beam if the two pulses did not overlap each other. The photon counting rate is about $260 \mathrm{kHz}$, which was almost the same as the sum of that from the two beams. Tuning the time delay between the two beams, the total counting rate of the Si-APD increased and reached a maximum when the two beams were temporally overlapped. This increase in the photon counting rate was considerablely caused by the ND-TPA. 
Similar to the principle of an optical autocorrelator, when the phase difference between the two beams was zero, ND-TPA got a maximum of $325 \mathrm{kHz}$. The full-width at half maximum of the curve is $14.2 \mathrm{ps}$, in good agreement with the laser pulse duration of 10 ps with $\mathrm{t}=\sqrt{2} \tau$, where $\mathrm{t}$ is the measured cross-correlation width and $\tau$ is the laser pulse duration.

(a)

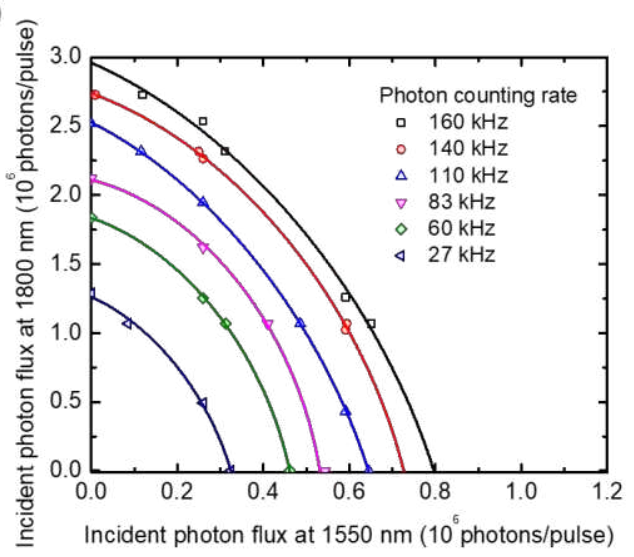

(b)

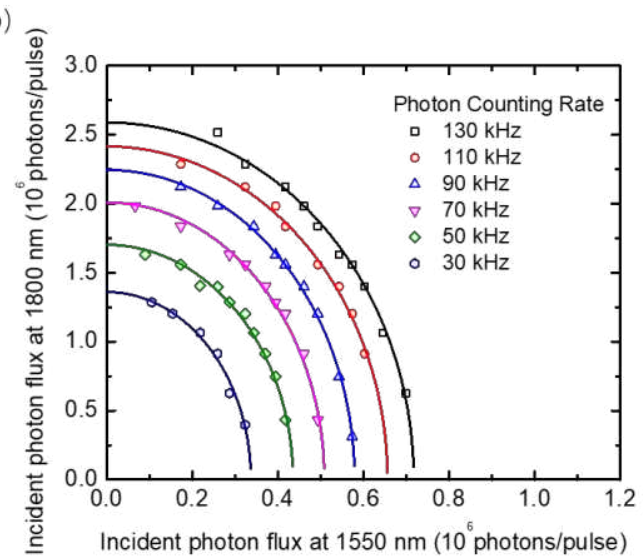

Fig.4 Incident photon flux of two beams at a certain counting rate under total TPA effect (a) and D-TPA effect alone (b).

Fixing the delay at zero, we could study the ND-TPA by comparing with D-TPA. We adjusted the incident photon fluxes of both beams to keep the photon counting rate at a certain value. In this case, the response of the Si-APD included both the ND-TPA contributed by the two beams and also the D-TPA from each beam individually. The photon counting rate follows

$$
C=\eta_{A B}\left\langle N_{A}\right\rangle\left\langle N_{B}\right\rangle+\eta_{A}\left\langle N_{A}\right\rangle^{2}+\eta_{B}\left\langle N_{B}\right\rangle^{2}
$$


where $\eta_{A B}, \eta_{A}, \eta_{B}$ represent the quantum detection efficiency by the ND-TPA effect, DTPA of Beam A, and D-TPA of Beam B respectively. And $\left\langle N_{A}\right\rangle$ and $\left\langle N_{B}\right\rangle$ are the photon fluxes of Beam A and Beam B, respectively. We adjusted the incident photon fluxes by the two VAs while keeping a certain total photon counting rate ranging from $27 \mathrm{kHz}$ to $160 \mathrm{kHz}$ as shown in Fig.4(a). By fitting the curves in Fig.4(a) according to Eq.(7), we get

$$
\begin{aligned}
& \eta_{A B}=(7.9 \pm 1.5) \times 10^{-16} \text { counts } \bullet \text { pulse } / \text { photon }^{2}, \\
& \eta_{A}=(3.3 \pm 0.1) \times 10^{-16} \text { counts } \bullet \text { pulse/photon }{ }^{2}, \\
& \eta_{B}=(4.3 \pm 0.26) \times 10^{-15} \text { counts } \bullet \text { pulse } / \text { photon }^{2} .
\end{aligned}
$$

Meanwhile, the D-TPA quantum detection efficiency were determined by recording the photon counting rates when the delay between the two beams was far from zero. The incident photon fluxes were recorded in the same way as in the ND-TPA measurement. All the curves are in a quarter ellipse shape obeying

$$
C^{\prime}=\eta_{A}\left\langle N_{A}\right\rangle^{2}+\eta_{B}\left\langle N_{B}\right\rangle^{2}
$$

And by fitting the curves in Fig.4(b), we get

$$
\begin{aligned}
& \eta_{A}=(3.2 \pm 0.2) \times 10^{-16} \text { counts } \bullet \text { pulse } / \text { photon }^{2}, \\
& \eta_{B}=(4.8 \pm 0.12) \times 10^{-15} \text { counts } \bullet \text { pulse } / \text { photon }^{2},
\end{aligned}
$$

which are almost the same as those in Eq.(8). We can conclude that the quantum detection efficiency of D-TPA keeps the same in the presence of ND-TPA. 
The spectral dependence of the TPA efficiency is expected to be different for indirect gap semiconductor as compared with that of the direct-gap semiconductors. According to Ref [25], the D-TPA coefficient has a maximum slightly above the indirect bandgap, at $\hbar \omega \cong(7 / 6) E_{g}$. Accordingly, the wavelength of the photon is at about $954 \mathrm{~nm}$. As the wavelength increases, the D-TPA efficiency decreases [23]. The D-TPA quantum detection efficiency at $1800 \mathrm{~nm}$ was measured to be more than one order smaller than that at $1550 \mathrm{~nm}$ in our experiment. Meanwhile, the ND-TPA efficiency was not equal to the D-TPA efficiency at either wavelength of $1800 \mathrm{~nm}$ and $1550 \mathrm{~nm}$. And there was no direct relation between the ND-TPA efficiency and the DTPA efficiencies of the two beams at different wavelengths.

\section{Conclusion}

In summary, we experimentally determined the specific contribution of D-TPA and ND-TPA effects at two different wavelengths of $1800 \mathrm{~nm}$ and $1550 \mathrm{~nm}$ for infrared light detection in the Si-APD single-photon detector at room temperature. The quantum detection efficiencies of D-TPA at $1800 \mathrm{~nm}$ and $1550 \mathrm{~nm}$ were measured to be about $3.2 \times 10^{-16}$ counts $\bullet$ pulse/photon ${ }^{2}$ and $4.8 \times 10^{-15}$ counts $\bullet$ pulse/photon ${ }^{2}$, respectively, and they kept the same in the presence of ND-TPA. Meanwhile the quantum detection efficiency of ND-TPA of $1550 \mathrm{~nm}$ and $1800 \mathrm{~nm}$ was measured to be $7.9 \times 10^{-16}$ counts•pulse/photon ${ }^{2}$. The TPA effect in photon detectors has shown great potential in many applications. Owing to the advantages of infrared photon detection based on TPA effect over the frequency upconversion scheme, we believe that high efficiency TPA 
quantum detectors of silicon-based optoelectronic devices will be developed in the near future for various applications. 


\section{Declarations}

\section{Availability of data and materials}

The datasets used and/or analyzed during the current study are available from the corresponding author on reasonable request.

\section{Competing interests}

The authors declare that they have no competing interests

\section{Funding}

National Key Research and Development Program (2018YFB0504400);

National Natural Science Foundation of China (11722431, 11774095, 11621404).

\section{Authors' contributions}

Guangjian $\mathrm{Xu}$, and Qucheng Miao conducted experiment and wrote the manuscript. Xinyi Ren and Ming Yan built the laser source. Haifeng Pan, Xiuliang Chen and Guang $\mathrm{Wu}$ designed the data collection system and analyzed the data. E $\mathrm{Wu}$ designed the experiment, analyzed the data and wrote the manuscript.

\section{Acknowledgements}

We thank Professor Kun Huang from East China Normal University for discussions. 


\section{References}

1. M. T. Rakher, L. Ma, O. Slattery, X. Tang, and K. Srinivasan (2010), Quantum transduction of telecommunications-band single photons from a quantum dot by frequency upconversion, Nat. Photonics 4, 786-791.

2. O. Kuzucu, F. N. C. Wong, S. Kurimura, and S. Tovstonog (2008), Joint Temporal Density Measurements for Two-Photon State Characterization,Phys. Rev. Lett. 101, 153602.

3. S. Liao, H. Yong, C. Liu, G. Shentu, D. Li, J. Lin, H. Dai, S. Zhao, B. Li, J. Guan, W. Chen, Y. Gong, Y. Li, Z. Lin, G. Pan. J. S. Pelc, M. M. Fejer, W. Zhang, W. Liu, J. Yin, J. Ren, X. Wang, Q. Zhang, C. Peng, and J. Pan (2017), Long-distance free-space quantum key distribution in daylight towards inter-satellite communication, Nat. Photonics 11, 509-513.

4. Y. Iwal, T. Honjo, K. Inoue, H. Kamada, Y. Nishida, O. Tadanaga, and M. Asobe (2009), Polarization-independent, differential-phase-shift, quantum-key distribution system using upconversion detectors, Opt. Lett. 34, 1606-1608.

5. N. Namekata, Y. Makino, and S. Inoue (2002), Single-photon detector for longdistance fiber-optic quantum key distribution, Opt. Lett. 27, 954-956.

6. D. A. Fishman, C. M. Cirloganu, S. Webster, L. A. Padilha, M. Monroe, D. J. Hagan, and E. W. Van Styland (2011), Sensitive mid-infrared detection in widebandgap semiconductors using extreme non-degenerate two-photon absorption, Nat. Photonics 5, 561-565. 
7. P. Apiratikul, and T. E. Murphy (2010), Background-suppressed ultrafast optical sampling using nondegenerate two-photon absorption in a GaAs photodiode, IEEE Photonics Technology Letters, 22, 212-214.

8. P. J. Maguire, L. P. Barry, T. Krug, W. H. Guo, J. O’Dowd, M. Lynch, A. L. Bradley, J. F. Donegan and H. Folliot (2006), Optical signal processing via two-photon absorption in a semiconductor microcavity for the next generation of high-speed optical communications network, J. Lightwave Technol. 24, 2683-2692.

9. J. T. Gomes, L. Delage, R. Baudoin, L. Grossard, L. Bouyeron, D. Ceus, F. Reynaud, H. Herrmann, and W. Sohler (2014), Laboratory demonstration of spatialcoherence analysis of a blackbody through an up-conversion interferometer, Phys. Rev. Lett. 112, 143904.

10. R. Tang, X. Li, W. Wu, H. Pan, H. Zeng, and E. Wu, "High efficiency frequency upconversion of photons carrying orbital angular momentum for a quantum information interface," Opt. Express 23(8), 9796-9802(2015).

11. A. Barh, C. Pedersen, and P. Tidemand-Lichtenberg (2017), Ultra-broadband midwave-IR upconversion detection, Opt. Lett. 42, 1504-1507.

12. X. Gu, K. Huang, Y. Li, H. Pan, E Wu, and H. Zeng (2010), Temporal and spectral control of single-photon frequency upconversion for pulsed radiation, Appl. Phys. Lett. 96, 131111.

13. L. Meng, A. Padhye, C. Pedersen, M. Ebrahim-Zaden, and P.J. Rodrigo (2019), SHG (532 nm)-induced spontaneous parametric downconversion noise in 1064-nm- 
pumped IR upconversion detectors, Opt. Lett. 44, 1670-1673.

14. J. Ma, X. Chen, H. Hu, H. Pan, E Wu, and H. Zeng (2016), Quantum detector tomography of a single-photon frequency upconversion detection system, Opt. Express 24, 20973-20981.

15. P. D. Olszak, C. M. Cirloganu, S. Webster, L. A. Padilha, S. Guha, L. P. Gonzalez, S. Krishnamurthy, D. J. Hagan, and E. W. Van Styland (2010), Spectral and temperature dependence of two-photon and free-carrier absorption in InSb, Phys. Rev. B. 82, 235207.

16. D. L. Boiko, A.V. Antonov, D. I. Kuritsyn, A. N. Yablonskiy, S. M. Sergeev, E. E. Orlova, and V. V. Vaks (2017), Mid-infrared two photon absorption sensitivity of commercial detectors, Appl. Phys. Lett. 111, 171102.

17. A. Hayat, P. Ginzburg, and M. Orenstein (2008), Infrared single-photon detection by two-photon absorption in silicon, Phys. Rev. B. 77, 125219.

18. G. Xu, X. Ren, Q. Miao, M. Yan, H. Pan, X. Chen, G. Wu, and E Wu (2019) Sensitive infrared photon counting detection by nondegenerate two-photon absorption in Si APD, IEEE Photon. Tech. Lett. 31, 1944-1947.

19. H. S. Pattanaik, M. Reichert, D. J. Hagan, and E. W. Van Styland (2016), Threedimensional IR imaging with uncooled GaN photodiodes using nondegenerate twophoton absorption. Opt. Express, 24, 1196.

20. M. Piccardo, N. A. Rubin, L. Meadowcroft, P. Chevalier, H. Yuan, J. Kimchi, and 
F. Capasso (2018), Mid-infrared two-photon absorption in an extended-wavelength InGaAs photodetector, Appl. Phys. Lett. 112(4), 041106.

21. B. Fix, J. Jaeck, B. Vest, M. Verdun, G. Beaudoin, I. Sagnes, J. L. Pelouard, and R. Haidar (2017), Nanostructured diode for infrared photodetection through nondegenerate two-photon absorption, Appl. Phys. Lett. 111(4), 041102.

22. J. L. Cheng, J. Rioux, and J. E. Sipe (2011), Full band structure calculation of twophoton indirect absorption in bulk silicon, Appl. Phys. Lett. 98(13), 131101.

23. A. D. Bristow, N. Rotenberg, and H. M. Van Driel (2007), Two-photon absorption and Kerr coefficients of silicon for 850-2200 nm, Appl. Phys. Lett. 90(19), 191104.

24. M. Sheik-Bahae, D. C. Hutchings, D. J. Hagan, and E. W. Van Stryland (1991), Dispersion of bound electronic nonlinear refraction in solids, IEEE J. Quantum Electron. 27(6), 1296-1309.

25. M. Dinu (2003), Dispersion of phonon-assisted nonresonant third-order nonlinearities. IEEE J. Quantum Electronics, 39(11), 1498-1503. 
Figures

(a)

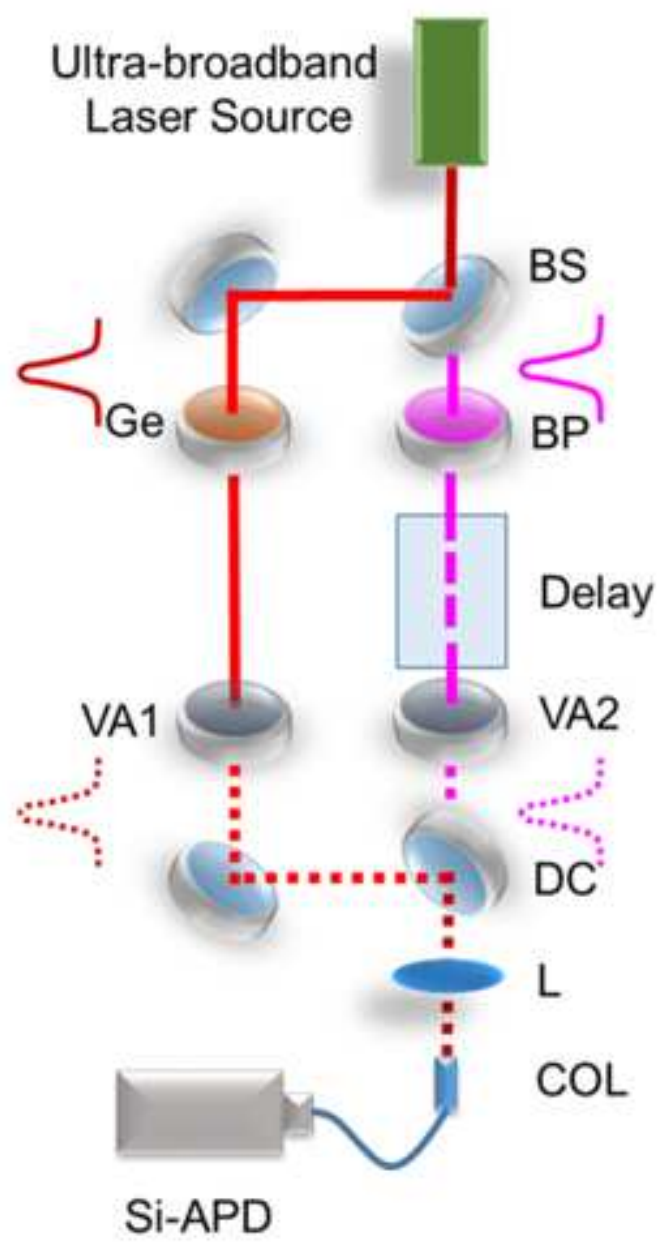

(b)

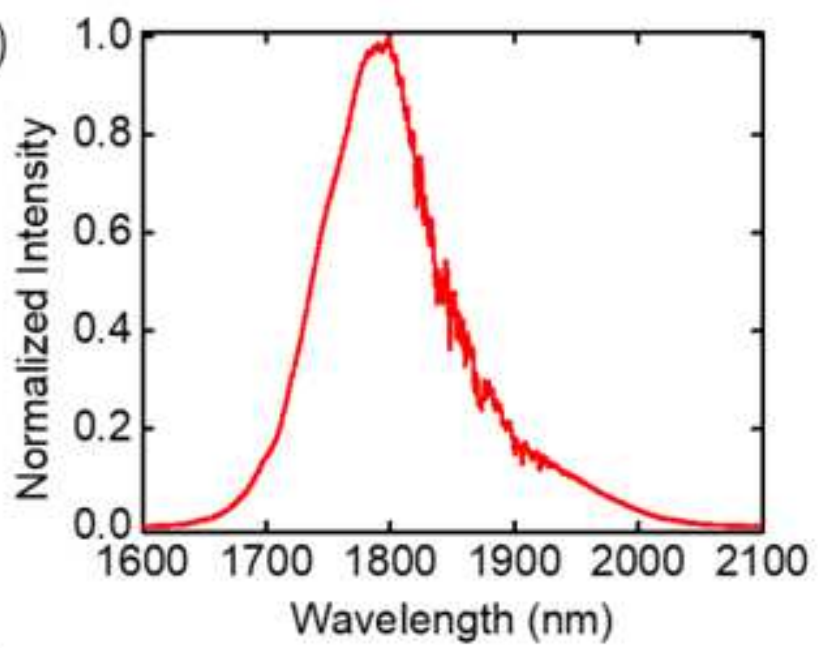

(c)

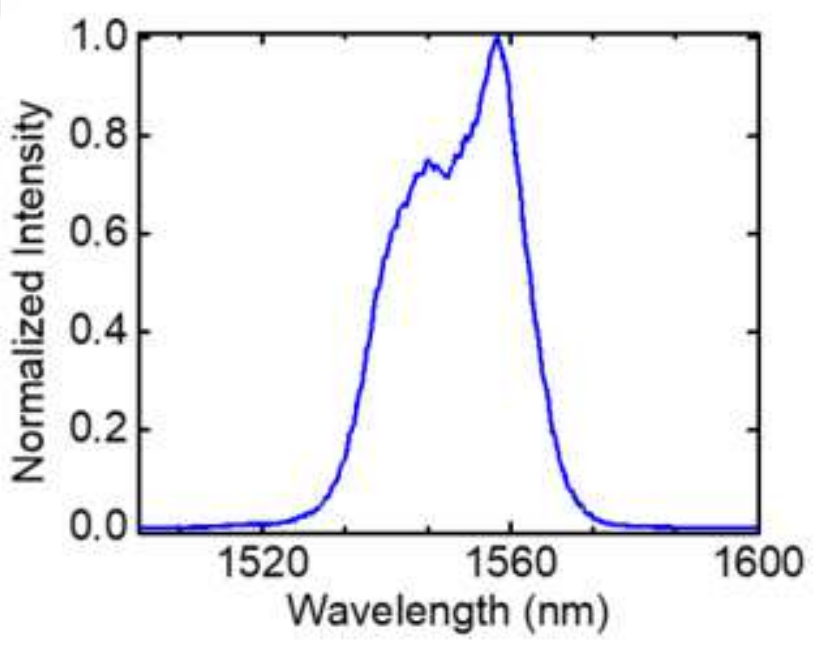

Figure 1

(a)Experimental setup. BS: Beamsplitter; Ge: Germanium window; BP: Bandpass filter at $1550 \mathrm{~nm}$; VA1,2: Variable intensity attenuators; DC: Dichroic mirror reflecting $1800 \mathrm{~nm}$ and transmitting $1550 \mathrm{~nm}$; L: Lens, COL: Fiber collimator; Si-APD: Single-photon detector based on silicon avalanche photodiode. (b) Spectrum of the Beam A at $1800 \mathrm{~nm}$. (c) Spectrum of the Beam B at $1550 \mathrm{~nm}$. 
(a)

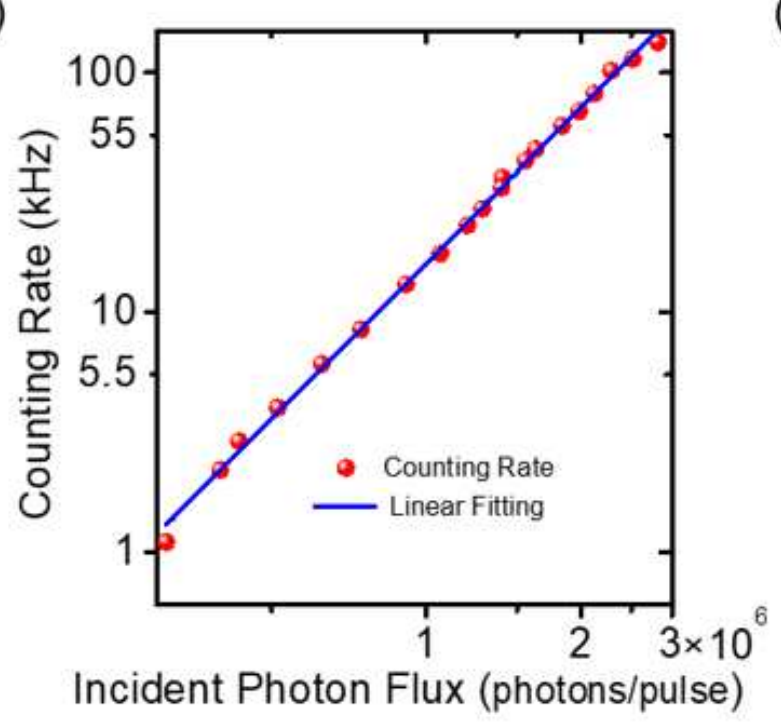

(b)

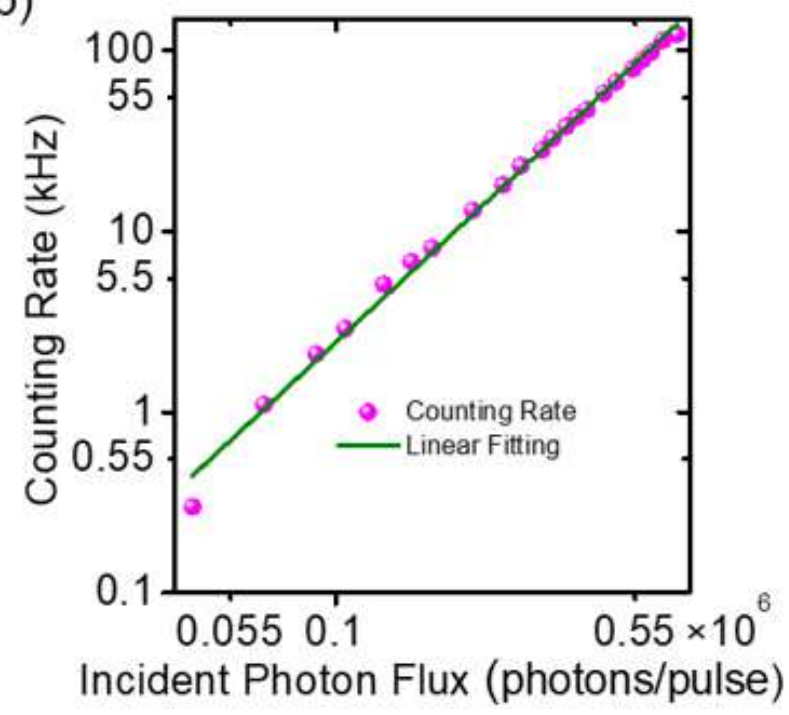

Figure 2

Photon-counting rate as a function of the incident photon flux with a single beam at $1800 \mathrm{~nm}(\mathrm{a})$ and $1550 \mathrm{~nm}(\mathrm{~b})$, respectively.

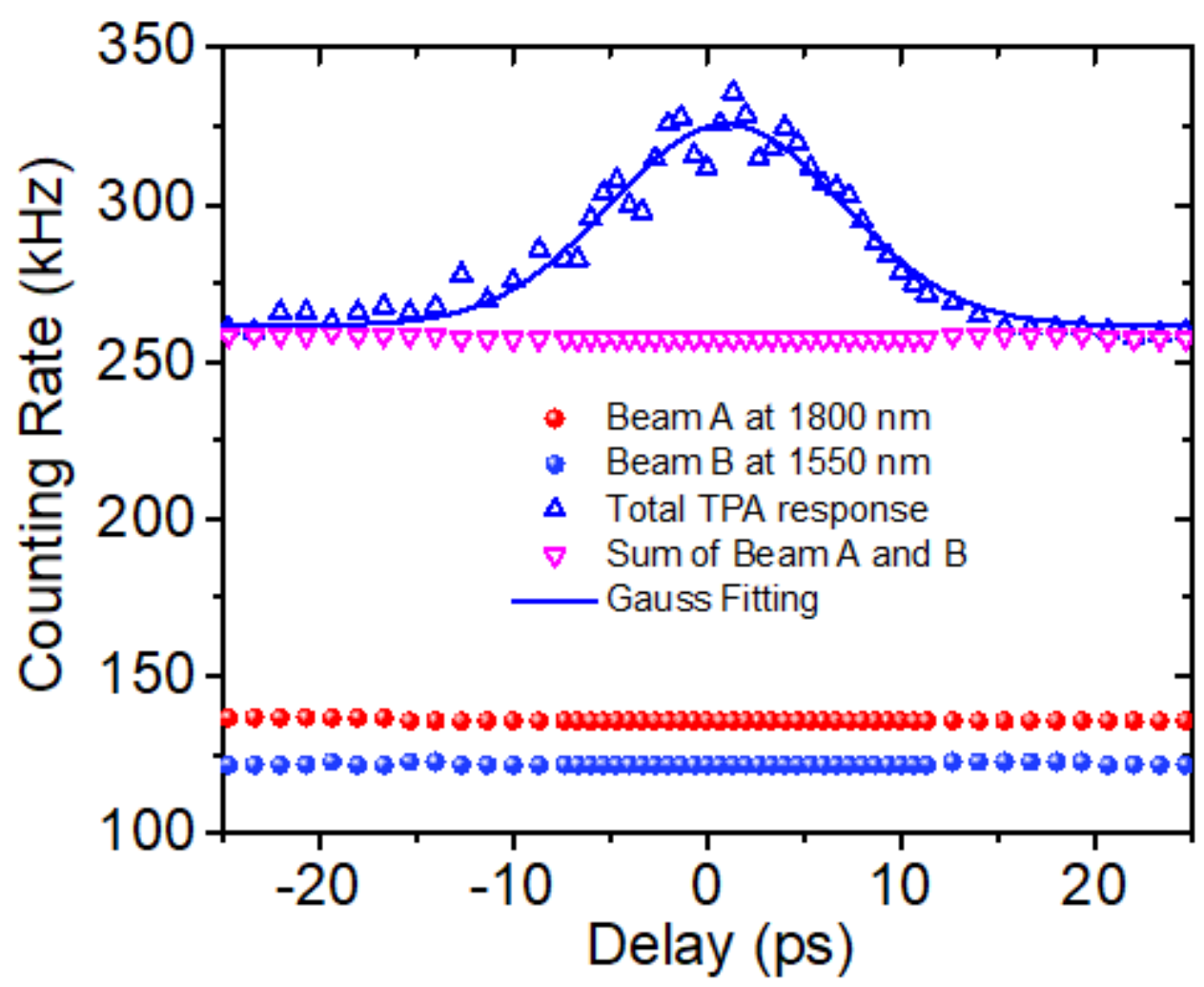

Figure 3 
Photon counting rate as a function of the relative delay between the two beams. The red spots and the blue spots are the counting rate of the D-TPA of $1800 \mathrm{~nm}$ and $1550 \mathrm{~nm}$ respectively. The pink triangle spots are the sum of the both D-TPA effect. And the blue triangle spots are the counting rate of the TPA of two beams.

(a)

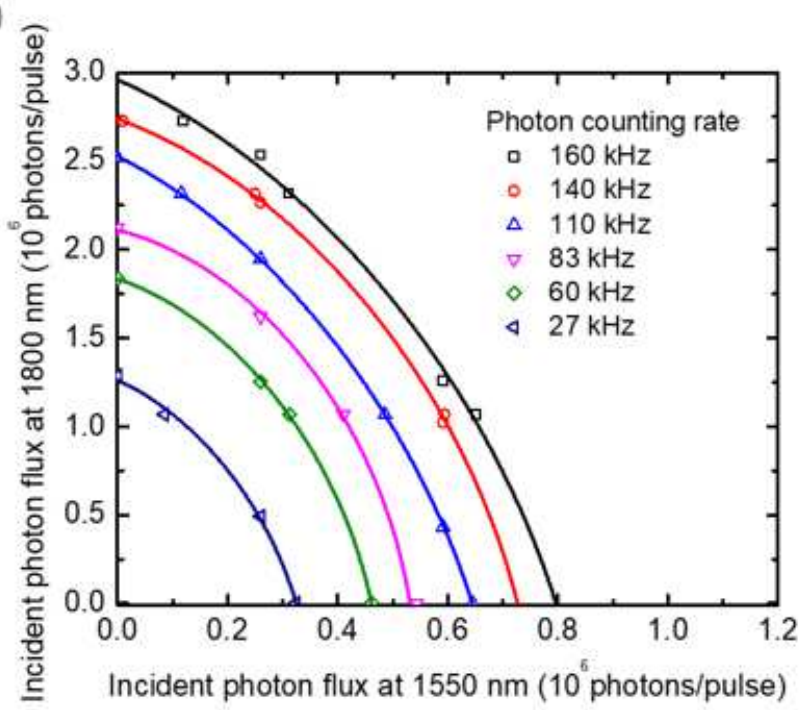

(b)

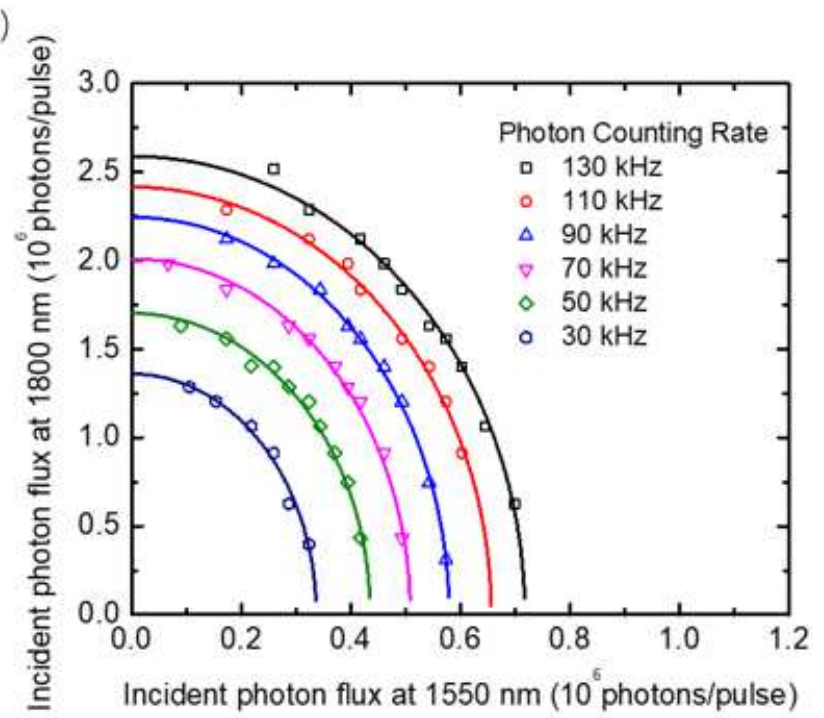

\section{Figure 4}

Incident photon flux of two beams at a certain counting rate under total TPA effect (a) and D-TPA effect alone (b). 